\title{
Generalized fractal Jensen and Jensen-Mercer inequalities for harmonic convex function with applications
}

\author{
Saad Ihsan Butt ${ }^{1 *}$, Praveen Agarwal ${ }^{2,3}$, Saba Yousaf ${ }^{1}$ and Juan L.G. Guirao ${ }^{4,5}$
}

\section{"Correspondence:}

saadihsanbutt@gmail.com

'Department of Mathematics, COMSATS University Islamabad, Lahore Campus, Pakistan Full list of author information is available at the end of the article

\section{Springer}

\begin{abstract}
In this paper, we present a generalized Jensen-type inequality for generalized harmonically convex function on the fractal sets, and a generalized Jensen-Mercer inequality involving local fractional integrals is obtained. Moreover, we establish some generalized Jensen-Mercer-type local fractional integral inequalities for harmonically convex function. Also, we obtain some generalized related results using these inequalities on the fractal space. Finally, we give applications of generalized means and probability density function.
\end{abstract}

MSC: Primary 26D10; secondary 26A51;25D15; 26A33

Keywords: Harmonic convex function; Jensen-type inequality; Jensen-Mercer inequality; Fractal space; Generalized harmonic convex function; Local fractional derivative; Local fractional integral

\section{Introduction}

In different fields of pure and applied mathematics, the convexity of functions has been used. Several new classes of convex functions and convex sets have been introduced and studied. Many researchers have derived the variety of new inequalities associated with these new classes of convex functions [1-3]. The harmonic set was introduced by Shi et al. [4]. It must be noted that the weighted harmonic mean is used to give a concept of the harmonic set. It has applications in the theory of electrical circuits and other fields of sciences. Harmonic convex functions are defined by using the weighted harmonic means, which have appeared as a significant and major generalization of convex functions. Several properties of harmonic convex functions have been investigated by İșcan [5], Dragomir [6], and Farid et al. in [7].

The definition of convex function is as follows.

Definition 1 A function $\Psi: I \subset \mathbb{R} \longrightarrow \mathbb{R}$ is said to be convex function on $I(\Psi \in K(I))$ if

$$
\Psi\left(\vartheta \zeta_{1}+(1-\vartheta) \zeta_{2}\right) \leq \vartheta \Psi\left(\zeta_{1}\right)+(1-\vartheta) \Psi\left(\zeta_{2}\right)
$$

holds for all $\zeta_{1}, \zeta_{2} \in I$ and $\vartheta \in[0,1]$.

(c) The Author(s) 2021. This article is licensed under a Creative Commons Attribution 4.0 International License, which permits use sharing, adaptation, distribution and reproduction in any medium or format, as long as you give appropriate credit to the original author(s) and the source, provide a link to the Creative Commons licence, and indicate if changes were made. The images or other third party material in this article are included in the article's Creative Commons licence, unless indicated otherwise in a credit line to the material. If material is not included in the article's Creative Commons licence and your intended use is not permitted by statutory regulation or exceeds the permitted use, you will need to obtain permission directly from the copyright holder. To view a copy of this licence, visit http://creativecommons.org/licenses/by/4.0/. 
Jensen's inequality (J. I) is the best-known result in the literature. The generalizations and improvements of Jensen's inequality have been a topic of supreme interest for researchers during the last few decades as evident from a large number of publications on the topic (see [8-10]). This inequality has also been used in various areas of science and technology to solve several problems, such as engineering, mathematical statistics, financial economics, and computer science. For some recent results, see [11-13].

Theorem 1 ([1]) If $\Psi \in K(I)$, then

$$
\Psi\left(\sum_{l=1}^{s} \gamma_{l} a_{l}\right) \leq \sum_{l=1}^{s} \gamma_{l} \Psi\left(a_{l}\right)
$$

for all $a_{l} \in I$ and $\gamma_{l} \in[0,1](l=1,2, \ldots, s)$ with $\sum_{l=1}^{s} \gamma_{l}=1$.

In [14], Mercer gave a new variant of (J. I) as follows.

Theorem 2 If $\Psi \in K(I)$ on the interval $[\mathfrak{m}, \mathfrak{M}]$, then

$$
\Psi\left(\mathfrak{M}+\mathfrak{m}-\sum_{l=1}^{s} \gamma_{l} a_{l}\right) \leq \Psi(\mathfrak{M})+\Psi(\mathfrak{m})-\sum_{l=1}^{s} \gamma_{l} \Psi\left(a_{l}\right)
$$

for all $a_{\iota} \in[\mathfrak{m}, \mathfrak{M}]$ and $\gamma_{\iota} \in[0,1](\iota=1,2, \ldots, s)$ with $\sum_{l=1}^{s} \gamma_{\iota}=1$

In [5], Iscan gave the definition of harmonic convexity as follows.

Definition 2 A function $\Psi: I \subset \mathbb{R} \backslash\{0\} \rightarrow \mathbb{R}$ is said to be harmonically convex on $I$ $(\Psi \in H K(I))$ if

$$
\Psi\left(\frac{\zeta_{1} \zeta_{2}}{\vartheta \zeta_{1}+(1-\vartheta) \zeta_{2}}\right) \leq \vartheta \Psi\left(\zeta_{2}\right)+(1-\vartheta) \Psi\left(\zeta_{1}\right)
$$

holds for all $\zeta_{1}, \zeta_{2} \in I, \zeta_{1}<\zeta_{2}$, and $\vartheta \in[0,1]$.

Dragomir established (J. I) for $\Psi \in H K(I)$ in [15] as follows.

Theorem 3 Let $I \subseteq(0, \infty)$ be an interval and $\Psi \in H K(I)$, then

$$
\Psi\left(\frac{1}{\sum_{l=1}^{s} \frac{\gamma_{l}}{a_{l}}}\right) \leq \sum_{i=1}^{s} \gamma_{l} \Psi\left(a_{l}\right)
$$

for all $a_{l} \in I$ and $\gamma_{l} \in[0,1],(l=1,2, \ldots, s)$ with $\sum_{l=1}^{s} \gamma_{l}=1$.

Very recently, Baloch et al. [16] presented a variant of (J. I) in the Mercer sense for $\Psi \in$ $H K(I)$ as follows. 
Theorem 4 If $\Psi \in H K(I)$ on the interval $I=[\mathfrak{m}, \mathfrak{M}] \subseteq(0, \infty)$, then the inequality

$$
\Psi\left(\frac{1}{\frac{1}{\mathfrak{M}}+\frac{1}{\mathfrak{m}}-\sum_{l=1}^{s} \frac{\gamma_{l}}{a_{l}}}\right) \leq \Psi(\mathfrak{M})+\Psi(\mathfrak{m})-\sum_{l=1}^{s} \gamma_{l} \Psi\left(a_{l}\right)
$$

holds for all $a_{l} \in[\mathfrak{m}, \mathfrak{M}]$ and $\gamma_{l} \in[0,1],(\iota=1,2, \ldots, s)$ with $\sum_{\imath=1}^{s} \gamma_{l}=1$.

Fractional calculus and local fractional calculus are very powerful tools in applied mathematics [17-21]. Yang [22] stated the definition of local fractional calculus. The local fractional calculus is used to deal with various non-differentiable problems that arise in a complex real-world phenomenon system. Local fractional functional analysis is a fully new area of mathematics and also a totally new view of the mathematical world. The theory of fractional calculus has played an important role in various fields of applied and pure sciences, for example, electricity, mechanics, biology, economics, chemistry, notably control theory, image processing, etc. The local fractional calculus is extremely practical and comprehensive in science and engineering for its real-world models (see [23]). Mandelbrot defined a fractal set as the one whose Hausdorff dimension strictly exceeds the topological dimension [24]. Many researchers contemplated the properties of a function on the fractal space and built numerous sorts of fractional calculus by utilizing distinctive approaches, see [25, 26]. Mo et al. [27] defined the generalized convex function on fractal sets $\mathbb{R}^{\varrho}(0<\varrho \leq 1)$ of real numbers and established generalized Jensen's and HermiteHadamard's inequalities for a generalized convex function in the concept of local fractional calculus. In (2017) Sun [28] introduced the concept of harmonic convex function on fractal sets $\mathbb{R}^{\varrho}(0<\varrho \leq 1)$ of real numbers and gave some Hermite-Hadamard inequalities for a generalized harmonic function $\left(\Psi \in G H K_{\varrho}(I)\right)$. In 2018, authors [29] worked on fractal integral inequalities for $\Psi \in G H K(I)$. Sun [30] in 2019 worked on generalization of some inequalities for $\left(\Psi \in G H K_{\varrho}(I)\right)$ on the fractal space. Recently, in 2020, Iftikhar et al. [31] gave several new Newton-type inequalities in a local fractional calculus scheme. Some recent results for a generalized harmonic convex function can be seen in [32].

\section{Preliminaries}

Using the Gao-Yang-Kang concept, recall the set $\mathbb{R}^{\varrho}$ to classify the definitions of local fractional derivative, local fractional integral (see [22]), and so on.

The theory of Yang's fractional sets [22] can be stated as follows.

For $0<\varrho \leq 1$, the $\varrho$-type set of element set is given below:

$\mathbb{Z}^{\varrho}$ : The set defined as the $\varrho$-type set of integer is $\left\{0^{\varrho}, \pm 1^{\varrho}, \pm 2^{\varrho}, \ldots, \pm n^{\varrho}, \ldots\right\}$.

$\mathbb{Q}^{\varrho}$ : The set defined as the $\varrho$-type set of rational numbers is $\left\{m^{\varrho}=(r / s)^{\varrho}: r, s \in \mathbb{Z}, s \neq 0\right\}$.

$\mathbb{J}^{\varrho}$ : The set defined as the $\varrho$-type set of irrational numbers is $\left\{m^{\varrho} \neq(r / s)^{\varrho}: r, s \in \mathbb{Z}, s \neq 0\right\}$.

$\mathbb{R}^{\varrho}$ : The set defined as the $\varrho$-type set of real numbers is $\mathbb{R}^{\varrho}=\mathbb{Q}^{\varrho} \cup J^{\varrho}$.

The following operations hold for $r^{\varrho}, s^{\varrho}$, and $t^{\varrho}$ belonging to the set $\mathbb{R}^{\varrho}$ of real line numbers:

(i) $r^{\varrho}+s^{\varrho}$ and $r^{\varrho} s^{\varrho}$ belong to the set $\mathbb{R}^{\varrho}$;

(ii) $r^{\varrho}+s^{\varrho}=s^{\varrho}+r^{\varrho}=(r+s)^{\varrho}=(s+r)^{\varrho}$;

(iii) $r^{\varrho}+\left(s^{\varrho}+t^{\varrho}\right)=(r+s)^{\varrho}+t^{\varrho}$;

(iv) $r^{\varrho} s^{\varrho}=s^{\varrho} r^{\varrho}=(r s)^{\varrho}=(s r)^{\varrho}$; 
(v) $r^{\varrho}\left(s^{\varrho} t^{\varrho}\right)=\left(r^{\varrho} s^{\varrho}\right) t^{\varrho}$;

(vi) $r^{\varrho}\left(s^{\varrho}+t^{\varrho}\right)=r^{\varrho} s^{\varrho}+r^{\varrho} t^{\varrho}$;

(vii) $r^{\varrho}+0^{\varrho}=0^{\varrho}+r^{\varrho}=r^{\varrho}$ and $r^{\varrho} 1^{\varrho}=1^{\varrho} r^{\varrho}=r^{\varrho}$.

Let us recall some basics of local fractional calculus on $\mathbb{R}^{\varrho}$.

Definition 3 ([22]) A non-differentiable function $\Psi: \mathbb{R} \longrightarrow \mathbb{R}^{\varrho}, \zeta \longrightarrow \Psi(\zeta)$ at $\zeta_{0}$ is continuous in a local fractional sense if, for any $\epsilon>0$, there exists $\delta>0$ such that

$$
\left|\Psi(\zeta)-\Psi\left(\zeta_{0}\right)\right|<\epsilon^{\varrho}
$$

holds for $\left|\zeta-\zeta_{0}\right|<\delta$, for all $\epsilon, \delta \in \mathbb{R}$. We denote $\Psi(\zeta) \in C_{\varrho}(\mathfrak{m}, \mathfrak{M})$ if $\Psi(\zeta)$ is local fractional continuous on the interval $(\mathfrak{m}, \mathfrak{M})$.

Definition 4 ([22]) The definition of local fractional derivative of $\Psi(\zeta)$ of order $\varrho$ at $\zeta=\zeta_{0}$ is

$$
\Psi^{(\varrho)}\left(\zeta_{0}\right)=\left.\frac{d^{\varrho} \Psi(\zeta)}{d \zeta^{\varrho}}\right|_{\zeta=\zeta_{0}}=\lim _{\zeta \rightarrow \zeta_{0}} \frac{\Delta^{\varrho}\left(\Psi(\zeta)-\Psi\left(\zeta_{0}\right)\right)}{\left(\zeta-\zeta_{0}\right)^{\varrho}}
$$

where $\Delta^{\varrho}\left(\Psi(\zeta)-\Psi\left(\zeta_{0}\right)\right) \cong \Gamma(1+\varrho)\left(\Psi(\zeta)-\Psi\left(\zeta_{0}\right)\right)$.

If for any $\zeta \in I \subseteq \mathbb{R}$ there exists $\Psi^{((p+1) \varrho)}(\zeta)=\overbrace{D_{\zeta}^{\varrho} \cdots D_{\zeta}^{\varrho}}^{p+1 \text { times }} \Psi(\zeta)$, then we say $\Psi \in D_{(p+1) \varrho}(I)$, where $p=0,1,2, \ldots$.

Definition 5 ([22]) If $\Psi \in C_{\varrho}[\mathfrak{m}, \mathfrak{M}]$, then the local fractional integral of $\Psi(\zeta)$ of order $\varrho$ is

$$
\begin{aligned}
\mathfrak{m}_{\mathfrak{M}}^{(\varrho)} \Psi(\zeta) & =\frac{1}{\Gamma(1+\varrho)} \int_{\mathfrak{m}}^{\mathfrak{M}} \Psi(w)(d w)^{\varrho} \\
& =\frac{1}{\Gamma(1+\varrho)} \lim _{\Delta w \rightarrow 0} \sum_{s=0}^{M-1} \Psi\left(w_{s}\right)\left(\Delta w_{s}\right)^{\varrho}
\end{aligned}
$$

with $\Delta w_{s}=w_{s+1}-w_{s}$ and $\Delta w=\max \left\{\Delta w_{s} \mid s=1,2, \ldots, M-1\right\}$, where $\left[w_{s}, w_{s+1}\right], s=$ $0,1, \ldots, M-1$, and $w_{0}=\mathfrak{m}<w_{1}<\cdots<w_{i}<\cdots<w_{M-1}<w_{M}=\mathfrak{M}$ is a partition of the interval $[\mathfrak{m}, \mathfrak{M}]$.

Here, it implies that ${ }_{\mathfrak{m}} I_{\mathfrak{M}}^{(\varrho)} \Psi(\zeta)=0$ if $\mathfrak{m}=\mathfrak{M}$ and ${ }_{\mathfrak{m}} I_{\mathfrak{M}}^{(\varrho)} \Psi(\zeta)=-\mathfrak{M} I_{\mathfrak{m}}^{(\varrho)} \Psi(\zeta)$ if $\mathfrak{m}<\mathfrak{M}$. If for any $\zeta \in[\mathfrak{m}, \mathfrak{M}]$ there exists $I_{\mathfrak{M}}^{(\varrho)} \Psi(\zeta)$, then we denote $\Psi(\zeta) \in I_{\zeta}^{(\varrho)}[\mathfrak{m}, \mathfrak{M}]$.

Lemma $1([22])$

(i) If $\Psi(\zeta)=\Upsilon^{(\varrho)}(\zeta) \in C_{\varrho}[\mathfrak{m}, \mathfrak{M}]$, then we have

$$
{ }_{\mathfrak{m}} I_{\mathfrak{M}}^{\varrho} \Psi(\zeta)=\Upsilon(\mathfrak{M})-\Upsilon(\mathfrak{m})
$$

(ii) If $\Psi(\zeta), \Upsilon(\zeta) \in D_{\varrho}[\mathfrak{m}, \mathfrak{M}]$ and $\Psi^{(\varrho)}(\zeta), \Upsilon^{(\varrho)}(\zeta) \in C_{\varrho}[\mathfrak{m}, \mathfrak{M}]$, then we have

$$
{ }_{\mathfrak{m}} I_{\mathfrak{M}}^{\varrho} \Psi(\zeta) \Upsilon^{(\varrho)}(\zeta)=\left.\Psi(\zeta) \Upsilon(\zeta)\right|_{\mathfrak{m}} ^{\mathfrak{M}}-{ }_{\mathfrak{m}} I_{\mathfrak{M}}^{\varrho} \Psi^{(\varrho)}(\zeta) \Upsilon(\zeta)
$$


Lemma 2 ([22])

$$
\frac{d^{\varrho} \zeta^{\zeta}}{d \zeta^{\varrho}}=\frac{\Gamma(1+\varsigma \varrho)}{\Gamma(1+(\varsigma-1) \varrho)} \zeta^{(\varsigma-1) \varrho}
$$

and

$$
\frac{1}{\Gamma(1+\varrho)} \int_{\mathfrak{m}}^{\mathfrak{M}} \zeta^{\varsigma \varrho}(d \zeta)^{\varrho}=\frac{\Gamma(1+\varsigma \varrho)}{\Gamma(1+(\varsigma+1) \varrho)}\left(\mathfrak{M}^{(\varsigma+1) \varrho}-\mathfrak{m}^{(\varsigma+1) \varrho}\right)
$$

while $\varsigma \in \mathbb{R}$.

The definition of a generalized harmonically convex function on fractal sets is as follows.

Definition 6 ([28]) A function $\Psi: I \subseteq \mathbb{R} \backslash\{0\} \longrightarrow \mathbb{R}^{\varrho}$ is said to be a generalized harmonic convex function on $I$ i.e. $\Psi \in G H K_{\varrho}(I)$ if

$$
\Psi\left(\frac{\zeta_{1} \zeta_{2}}{\vartheta \zeta_{1}+(1-\vartheta) \zeta_{2}}\right) \leq(1-\vartheta)^{\varrho} \Psi\left(\zeta_{1}\right)+\vartheta^{\varrho} \Psi\left(\zeta_{2}\right)
$$

holds for all $\zeta_{1}, \zeta_{2} \in I, \zeta_{1}<\zeta_{2}$, and $\vartheta \in[0,1]$.

The main aim of this paper is to present the generalized Jensen inequality (G. J. I) and generalized Jensen-Mercer inequality (G. J. M. I) for the class of functions $G H K_{\varrho}(I)$ on the fractal space. Moreover, we establish an improvement and generalization of some JensenMercer-type inequalities for harmonically convex function via local fractional integrals. Also, we obtain some generalized related results on a fractal space. Finally, we present some resulting applications to special means and probability density function.

\section{Generalized Jensen's and Jensen-Mercer inequalities for $G H K_{\varrho}(l)$ in the fractal sense}

In this section, we first present the (G. J. I) and establish (G. J. M. I) for $\Psi \in G H K_{\varrho}(I)$ via fractional integrals. In order to prove (G. J. M. I), we need the main identity later in this section.

Theorem 5 (G.J.I) Let $I \subseteq(0, \infty)$ be an interval and $\Psi \in G H K_{\varrho}(I)$, then

$$
\Psi\left(\frac{1}{\sum_{l=1}^{s} \frac{\gamma_{l}}{a_{l}}}\right) \leq \sum_{l=1}^{s} \gamma_{l}^{\varrho} \Psi\left(a_{l}\right)
$$

holds for all $a_{\iota} \in I$ and $\gamma_{\iota} \in[0,1],(\iota=1,2, \ldots, s)$ with $\sum_{l=1}^{s} \gamma_{\iota}=1$.

Proof The inequality is clearly true for $s=2$. Suppose that for $s=k$ the inequality is also true. Then, for any $a_{1}, a_{2}, \ldots, a_{k} \in I$ and $\omega_{l}>0, l=1,2, \ldots, k$ with $\sum_{l=1}^{k} \omega_{l}=1$, we have

$$
\Psi\left(\frac{1}{\sum_{l=1}^{k} \frac{\omega_{l}}{a_{l}}}\right) \leq \sum_{l=1}^{k} \omega_{l}^{\varrho} \Psi\left(a_{l}\right) .
$$


If $a_{1}, a_{2}, \ldots, a_{k}, a_{(k+1)} \in I$ and $\gamma_{l}>0$ for $\iota=1,2, \ldots, k+1$ with $\sum_{l=1}^{k+1} \gamma_{l}=1$, then one sets up

$$
\omega_{l}=\frac{\gamma_{l}}{1-\gamma_{k+1}}
$$

for all $\iota=1,2, \ldots, k$. It is easy to see that $\sum_{l=1}^{k} \omega_{l}=1$. Thus,

$$
\begin{aligned}
& \Psi\left(\frac{1}{\sum_{l=1}^{k+1} \frac{\gamma_{l}}{a_{l}}}\right)=\Psi\left(\frac{1}{\left(1-\gamma_{k+1}\right) \frac{\frac{\gamma_{1}}{a_{1}}+\frac{\gamma_{2}}{a_{2}}+\cdots+\frac{\gamma_{k}}{a_{k}}}{1-\gamma_{k+1}}+\frac{\gamma_{k+1}}{a_{k+1}}}\right) \\
& \leq\left(1-\gamma_{k+1}\right)^{\varrho} \Psi\left(\frac{1-\gamma_{k+1}}{\frac{\gamma_{1}}{a_{1}}+\frac{\gamma_{2}}{a_{2}}+\cdots+\frac{\gamma_{k}}{a_{k+1}}}\right)+\gamma_{k+1}^{\varrho} \Psi\left(a_{k+1}\right) \\
& =\left(1-\gamma_{k+1}\right)^{\varrho} \Psi\left(\frac{1}{\omega_{1} \frac{1}{a_{1}}+\omega_{2} \frac{1}{a_{2}}+\cdots+\omega_{k} \frac{1}{a_{k}}}\right)+\gamma_{k+1}^{\varrho} \Psi\left(a_{k+1}\right) \\
& \leq\left(1-\gamma_{k+1}\right)^{\varrho}\left[\omega_{1}^{\varrho} \Psi\left(a_{1}\right)+\omega_{2}^{\varrho} \Psi\left(a_{2}\right)+\cdots+\omega_{k}^{\varrho} \Psi\left(a_{k}\right)\right]+\gamma_{k+1}^{\varrho} \Psi\left(a_{k+1}\right) \\
& =\left(1-\gamma_{k+1}\right)^{\varrho}\left[\left(\frac{\gamma_{1}}{1-\gamma_{k+1}}\right)^{\varrho} \Psi\left(a_{1}\right)+\cdots+\left(\frac{\gamma_{k}}{1-\gamma_{k+1}}\right)^{\varrho} \Psi\left(a_{k}\right)\right] \\
& +\gamma_{k+1}^{\varrho} \Psi\left(a_{k+1}\right) \\
& =\sum_{l=1}^{k+1} \gamma_{l}^{\varrho} \Psi\left(a_{l}\right)
\end{aligned}
$$

So, the mathematical induction gives the proof of Theorem 5 .

Remark 1 If we take $\varrho=1$ in Theorem 5 , then it gives inequality (1) proved by Dragomir.

The main lemma for $\Psi \in G H K_{\varrho}(I)$ pertaining local fractional integrals is as follows.

Lemma 3 Let $I=[\mathfrak{m}, \mathfrak{M}] \subseteq(0, \infty)$ be an interval, $\left\{a_{l}\right\}_{l=1}^{s} \in I$ be a finite positive increasing sequence, and $\Psi \in G H K_{\varrho}(I)$ on the interval $[\mathfrak{m}, \mathfrak{M}]$, then

$$
\Psi\left(\frac{1}{\frac{1}{\mathfrak{M}}+\frac{1}{\mathfrak{m}}-\frac{1}{a_{l}}}\right) \leq \Psi(\mathfrak{M})+\Psi(\mathfrak{m})-\Psi\left(a_{l}\right)
$$

holds for all $1 \leq \imath \leq s$.

Proof Let $\frac{1}{b_{l}}=\frac{1}{\mathfrak{M}}+\frac{1}{\mathfrak{m}}+\frac{1}{a_{l}}$. Then $\frac{1}{b_{l}}+\frac{1}{a_{l}}=\frac{1}{\mathfrak{m}}+\frac{1}{\mathfrak{M}}$ so that the pairs $\mathfrak{m}, \mathfrak{M}$ and $a_{l}, b_{l}$ possess the same harmonic mean. Since that is the case, there exists $\vartheta$ such that

$$
\begin{aligned}
& a_{l}=\frac{\mathfrak{m} \mathfrak{M}}{\vartheta \mathfrak{m}+(1-\vartheta) \mathfrak{M}}, \\
& b_{l}=\frac{\mathfrak{m} \mathfrak{M}}{(1-\vartheta) \mathfrak{m}+\vartheta \mathfrak{M}},
\end{aligned}
$$


where $0 \leq \vartheta \leq 1$ and $1 \leq \imath \leq s$. Hence, applying generalized harmonic convexity of $\Psi$, we get

$$
\begin{aligned}
\Psi\left(b_{l}\right) & =\Psi\left(\frac{\mathfrak{m} \mathfrak{M}}{(1-\vartheta) \mathfrak{m}+\vartheta \mathfrak{M}}\right) \\
& \leq \vartheta^{\varrho} \Psi(\mathfrak{m})+(1-\vartheta)^{\varrho} \Psi(\mathfrak{M}) \\
& =\Psi(\mathfrak{M})+\Psi(\mathfrak{m})-\left[(1-\vartheta)^{\varrho} \Psi(\mathfrak{m})+\vartheta^{\varrho} \Psi(\mathfrak{M})\right] \\
& \leq \Psi(\mathfrak{M})+\Psi(\mathfrak{m})-\Psi\left(\frac{\mathfrak{m} \mathfrak{M}}{\vartheta \mathfrak{m}+(1-\vartheta) \mathfrak{M}}\right) \\
& =\Psi(\mathfrak{M})+\Psi(\mathfrak{m})-\Psi\left(a_{\imath}\right)
\end{aligned}
$$

and $\frac{1}{b_{l}}=\frac{1}{\mathfrak{M}}+\frac{1}{\mathfrak{m}}+\frac{1}{a_{l}}$, which concludes the proof of the lemma.

By using the (G. J.I), (G. J. M. I) for $\Psi \in G H K_{\varrho}(I)$ can be represented in a local fractional integral as follows.

Theorem 6 ((G. J. M. I)) Let $I=[\mathfrak{m}, \mathfrak{M}] \subseteq(0, \infty)$ be an interval and $\Psi \in G H K_{\varrho}(I)$, then the inequality

$$
\Psi\left(\frac{1}{\frac{1}{\mathfrak{M}}+\frac{1}{\mathfrak{m}}-\sum_{l=1}^{s} \frac{\gamma_{l}}{a_{l}}}\right) \leq \Psi(\mathfrak{M})+\Psi(\mathfrak{m})-\sum_{i=1}^{s} \gamma_{l}^{\varrho} \Psi\left(a_{l}\right)
$$

holds for any finite positive sequence $\left\{a_{l}\right\}_{l=1}^{s} \in I$ and $\gamma_{l} \in[0,1](l=1,2, \ldots, s)$ with $\sum_{l=1}^{s} \gamma_{l}=1$.

Proof It follows from Theorem 5 and Lemma 3 together with the generalized harmonic convexity of $\Psi$ on the interval $[\mathfrak{m}, \mathfrak{M}]$ that

$$
\begin{aligned}
\Psi\left(\frac{1}{\frac{1}{\mathfrak{M}}+\frac{1}{\mathfrak{m}}-\sum_{l=1}^{s} \frac{\gamma_{l}}{a_{l}}}\right) & =\Psi\left(\frac{1}{\sum_{l=1}^{s} \gamma_{l}\left(\frac{1}{\mathfrak{M}}+\frac{1}{\mathfrak{m}}-\frac{1}{a_{l}}\right)}\right) \\
& \leq \sum_{l=1}^{s} \gamma_{l}^{\varrho} \Psi\left(\frac{1}{\frac{1}{\mathfrak{M}}+\frac{1}{\mathfrak{m}}-\frac{1}{a_{l}}}\right) \\
& \leq \sum_{l=1}^{s} \gamma_{l}^{\varrho} \Psi(\mathfrak{M})+\sum_{l=1}^{s} \gamma_{l}^{\varrho} \Psi(\mathfrak{m})-\sum_{l=1}^{s} \gamma_{l}^{\varrho} \Psi\left(a_{l}\right) \\
& =\Psi(\mathfrak{M})+\Psi(\mathfrak{m})-\sum_{l=1}^{s} \gamma_{l}^{\varrho} \Psi\left(a_{l}\right),
\end{aligned}
$$

and this concludes the proof.

Remark 2 If we take $\varrho=1$ in Theorem 6, then it gives [[16], Theorem 2.4] and also see Theorem 4. 


\section{Improvement and generalization of some (G. J. M)-type inequalities for local fractional integrals}

Based on Lemma 3, some (G. J. M)-type inequalities can be represented pertaining local fractional integral forms as follows.

Theorem 7 Assume that $\Psi \in G H K_{\varrho}(I)$ on the interval $I=[\mathfrak{m}, \mathfrak{M}] \subset(0, \infty)$, then the following inequality for local fractional integrals holds:

$$
\begin{aligned}
\Psi\left(\frac{1}{\frac{1}{\mathfrak{M}}+\frac{1}{\mathfrak{m}}-\frac{1}{\bar{a}}}\right) & \leq \sum_{l=1}^{s} \gamma_{l}^{\varrho} \Psi\left(\frac{1}{\frac{1}{\mathfrak{M}}+\frac{1}{\mathfrak{m}}-\left(\frac{1-\vartheta}{\bar{a}}+\frac{\vartheta}{a_{l}}\right)}\right) \\
& \leq \Psi(\mathfrak{M})+\Psi(\mathfrak{m})-\sum_{l=1}^{s} \gamma_{l}^{\varrho} \Psi\left(a_{l}\right)
\end{aligned}
$$

for all $a_{l} \in[\mathfrak{m}, \mathfrak{M}]$ and $\gamma_{l} \in[0,1](l=1,2, \ldots, s)$ with $\sum_{l=1}^{s} \gamma_{l}=1$, where $\frac{1}{\bar{a}}=\sum_{l=1}^{s} \gamma_{l} \frac{1}{a_{l}}$.

Proof Since $\Psi \in G H K_{\varrho}(I)$, we have

$$
\begin{aligned}
\sum_{l=1}^{s} \gamma_{l}^{\varrho} \Psi\left(\frac{1}{\frac{1}{\mathfrak{M}}+\frac{1}{\mathfrak{m}}-\left(\frac{1-\vartheta}{\bar{a}}+\frac{\vartheta}{a_{l}}\right)}\right) & \geq \Psi\left(\frac{1}{\sum_{l=1}^{s} \gamma_{l}\left(\frac{1}{\mathfrak{M}}+\frac{1}{\mathfrak{m}}-\left(\frac{1-\vartheta}{\bar{a}}+\frac{\vartheta}{a_{l}}\right)\right)}\right) \\
& =\Psi\left(\frac{1}{\frac{1}{\mathfrak{M}}+\frac{1}{\mathfrak{m}}-\frac{1}{\bar{a}}+\frac{\vartheta}{\bar{a}}-\sum_{l=1}^{s} \gamma_{l} \frac{\vartheta}{a_{l}}}\right) \\
& =\Psi\left(\frac{1}{\frac{1}{\mathfrak{M}}+\frac{1}{\mathfrak{m}}-\frac{1}{\bar{a}}}\right) .
\end{aligned}
$$

On the other hand,

$$
\begin{aligned}
\sum_{l=1}^{s} \gamma_{l}^{\varrho} \Psi\left(\frac{1}{\frac{1}{\mathfrak{M}}+\frac{1}{\mathfrak{m}}-\left(\frac{1-\vartheta}{\bar{a}}+\frac{\vartheta}{a_{l}}\right)}\right) \\
=\sum_{i=1}^{s} \gamma_{l}^{\varrho} \Psi\left(\frac{1}{(1-\vartheta)\left(\frac{1}{\mathfrak{M}}+\frac{1}{\mathfrak{m}}-\frac{1}{\bar{a}}\right)+\vartheta\left(\frac{1}{\mathfrak{M}}+\frac{1}{\mathfrak{m}}-\frac{1}{a_{l}}\right)}\right) \\
\leq \sum_{l=1}^{s} \gamma_{l}^{\varrho}\left[(1-\vartheta)^{\varrho} \Psi\left(\frac{1}{\frac{1}{\mathfrak{M}}+\frac{1}{\mathfrak{m}}-\frac{1}{\bar{a}}}\right)+\vartheta^{\varrho} \Psi\left(\frac{1}{\frac{1}{\mathfrak{M}}+\frac{1}{\mathfrak{m}}-\frac{1}{a_{l}}}\right)\right] \\
\leq \sum_{l=1}^{s} \gamma_{l}^{\varrho}\left[(1-\vartheta)^{\varrho}\left(\Psi(\mathfrak{M})+\Psi(\mathfrak{m})-\sum_{j=1}^{s} \gamma_{j}^{\varrho} \Psi\left(a_{j}\right)\right)\right. \\
\left.\quad+\vartheta^{\varrho}\left(\Psi(\mathfrak{M})+\Psi(\mathfrak{m})-\Psi\left(a_{l}\right)\right)\right] \\
=\Psi(\mathfrak{M})+\Psi(\mathfrak{m})-\sum_{l=1}^{s} \gamma_{l}^{\varrho} \Psi\left(a_{l}\right) .
\end{aligned}
$$

Combining inequalities (7) and (8), we get (6).

Remark 3 If we take $\varrho=1$ in Theorem 7, then it gives [[33], Theorem 2.3]. 
Corollary 1 Let all assumptions of Theorem 7 hold and $\Psi(x) \in I_{x}^{(\varrho)}[\mathfrak{m}, \mathfrak{M}]$, then

$$
\begin{aligned}
\Psi\left(\frac{1}{\frac{1}{\mathfrak{M}}+\frac{1}{\mathfrak{m}}-\frac{1}{\bar{a}}}\right) & \leq \sum_{i=1}^{s} \frac{\gamma_{l}^{\varrho} \Gamma(1+\varrho)}{\left(\frac{1}{a_{l}}-\frac{1}{\bar{a}}\right) \varrho} \frac{1}{\frac{1}{\mathfrak{M}}+\frac{1}{\mathfrak{m}}-\frac{1}{\bar{a}}} \frac{I^{(\varrho)}}{\frac{1}{\mathfrak{M}}+\frac{1}{\mathfrak{m}}-\frac{1}{a_{l}}} \frac{\Psi(x)}{x^{2 \varrho}} \\
& \leq \Psi(\mathfrak{M})+\Psi(\mathfrak{m})-\sum_{l=1}^{s} \gamma_{l}^{\varrho} \Psi\left(a_{l}\right) .
\end{aligned}
$$

Proof Multiplying by $\frac{1}{\Gamma(1+\varrho)}$ on both sides of (6) and then integrating w.r.t $\vartheta$ over $[0,1]$, we get (9). Here, we used the fact

$$
\begin{aligned}
& \frac{1}{\Gamma(1+\varrho)} \int_{0}^{1} \Psi\left(\frac{1}{\frac{1}{\mathfrak{M}}+\frac{1}{\mathfrak{m}}-\left(\frac{1-\vartheta}{\bar{a}}+\frac{\vartheta}{a_{l}}\right)}\right)(d \vartheta)^{\varrho} \\
& \quad=\frac{1}{\Gamma(1+\varrho)} \int_{0}^{1} \Psi\left(\frac{1}{(1-\vartheta) \Psi\left(\frac{1}{\mathfrak{M}}+\frac{1}{\mathfrak{m}}-\frac{1}{\bar{a}}\right)+\vartheta \Psi\left(\frac{1}{\mathfrak{M}}+\frac{1}{\mathfrak{m}}-\frac{1}{a_{l}}\right)}\right)(d \vartheta)^{\varrho} \\
& \quad=\frac{1}{\left(\frac{1}{a_{l}}-\frac{1}{\bar{a}}\right) \varrho}\left[\frac{1}{\Gamma(1+\varrho)} \int_{\frac{1}{\frac{1}{\mathfrak{M}}+\frac{1}{\mathfrak{m}}-\frac{1}{a_{l}}}}^{\frac{1}{\mathfrak{m}}-\frac{1}{\bar{a}}} \frac{\Psi(t)}{t^{2 \varrho}}(d t)^{\varrho}\right] \\
& \quad=\frac{1}{\left(\frac{1}{a_{l}}-\frac{1}{\bar{a}}\right) \varrho} \frac{1}{\frac{1}{\mathfrak{M}+\frac{1}{\mathfrak{m}}-\frac{1}{\bar{a}}}} I_{\frac{1}{\mathfrak{M}}+\frac{1}{\mathfrak{m}}-\frac{1}{a_{l}}}^{(\varrho)} \frac{\Psi(x)}{x^{2 \varrho}} .
\end{aligned}
$$

Remark 4 If we take $\varrho=1$ in Corollary 1 , then it gives [[33], Corollary 2.4] and if put $\varrho=1$, $s=2, \gamma_{1}=\gamma_{2}=\frac{1}{2}, a_{1}=a$, and $a_{2}=b$ in Corollary 1 , then it gives [[33], Theorem 2.1].

Now, we present a more precise estimate in the following theorem.

Theorem 8 Assume that $\Psi \in G H K_{\varrho}(I)$ on the interval $I=[\mathfrak{m}, \mathfrak{M}] \subset(0, \infty)$, then the following inequality for local fractional integrals holds:

$$
\begin{aligned}
\Psi\left(\frac{1}{\frac{1}{\mathfrak{M}}+\frac{1}{\mathfrak{m}}-\frac{1}{\bar{a}}}\right) & \leq \sum_{i=1}^{s} \gamma_{l}^{\varrho} \Psi\left(\frac{1}{\frac{1}{\mathfrak{M}}+\frac{1}{\mathfrak{m}}-\frac{1}{2}\left(\frac{1}{\bar{a}}+\frac{1}{a_{l}}\right)}\right) \\
& \leq \sum_{l=1}^{s} \frac{\gamma_{l}^{\varrho} \Gamma(1+\varrho)}{\left(\frac{1}{a_{l}}-\frac{1}{\bar{a}}\right) \varrho} \frac{1}{\frac{1}{\mathfrak{M}}+\frac{1}{\mathfrak{m}}-\frac{1}{\bar{a}}} I^{(\varrho)} \frac{1}{\frac{1}{\mathfrak{M}}+\frac{1}{\mathfrak{m}}-\frac{1}{a_{l}}} \frac{\Psi(x)}{x^{2 \varrho}} \\
& \leq \Psi(\mathfrak{M})+\Psi(\mathfrak{m})-\sum_{l=1}^{s} \gamma_{l}^{\varrho} \Psi\left(a_{l}\right)
\end{aligned}
$$

for all $a_{l} \in[\mathfrak{m}, \mathfrak{M}]$ and $\gamma_{l} \in[0,1](l=1,2, \ldots, s)$ with $\sum_{l=1}^{s} \gamma_{l}=1$, where $\frac{1}{\overline{\bar{a}}}=\sum_{l=1}^{s} \gamma_{l} \frac{1}{a_{l}}$

Proof As $\Psi \in G H K_{\varrho}(I)$, then for any $a_{1}, b_{1} \in[\mathfrak{m}, \mathfrak{M}]$ and $\vartheta \in[0,1]$, we have

$$
\begin{aligned}
\Psi\left(\frac{2 a_{1} b_{1}}{a_{1}+b_{1}}\right) & =\Psi\left(\frac{1}{\frac{1}{2}\left(\frac{\vartheta}{a_{1}}+\frac{1-\vartheta}{b_{1}}+\frac{1-\vartheta}{a_{1}}+\frac{\vartheta}{b_{1}}\right)}\right) \\
& \leq \frac{1}{2^{\varrho}}\left[\Psi\left(\frac{1}{\frac{\vartheta}{a_{1}}+\frac{1-\vartheta}{b_{1}}}\right)+\Psi\left(\frac{1}{\frac{1-\vartheta}{a_{1}}+\frac{\vartheta}{b_{1}}}\right)\right]
\end{aligned}
$$




$$
\leq \frac{\Psi\left(a_{1}\right)+\Psi\left(b_{1}\right)}{2^{\varrho}} .
$$

Writing $a_{1}=\frac{1}{\frac{1}{\mathfrak{M}}+\frac{1}{\mathfrak{m}}-\frac{1}{a}}$ and $b_{1}=\frac{1}{\frac{1}{\mathfrak{M}}+\frac{1}{\mathfrak{m}}-\frac{1}{b}}$ for $a, b \in[\mathfrak{m}, \mathfrak{M}]$, we get

$$
\begin{aligned}
\Psi\left(\frac{1}{\frac{1}{\mathfrak{M}}+\frac{1}{\mathfrak{m}}-\frac{a+b}{2 a b}}\right) & \leq \frac{1}{2^{\varrho}}\left[\Psi\left(\frac{1}{\frac{1}{\mathfrak{M}}+\frac{1}{\mathfrak{m}}-\left(\frac{\vartheta}{a}+\frac{1-\vartheta}{b}\right)}\right)+\Psi\left(\frac{1}{\frac{1}{\mathfrak{M}}+\frac{1}{\mathfrak{m}}-\left(\frac{1-\vartheta}{a}+\frac{\vartheta}{b}\right)}\right)\right] \\
& \leq \frac{1}{2^{\varrho}}\left[\Psi\left(\frac{1}{\frac{1}{\mathfrak{M}}+\frac{1}{\mathfrak{m}}-\frac{1}{a}}\right)+\Psi\left(\frac{1}{\frac{1}{\mathfrak{M}}+\frac{1}{\mathfrak{m}}-\frac{1}{b}}\right)\right] .
\end{aligned}
$$

Multiplying by $\frac{1}{\Gamma(1+\varrho)}$ on both sides of the above equation, then integrating w.r.t $\vartheta$ over $[0,1]$ and using fact that

$$
\begin{aligned}
& \frac{1}{\Gamma(1+\varrho)} \int_{0}^{1} \Psi\left(\frac{1}{\frac{1}{\mathfrak{M}}+\frac{1}{\mathfrak{m}}-\left(\frac{\vartheta}{a}+\frac{1-\vartheta}{b}\right)}\right)(d \vartheta)^{\varrho} \\
& \quad=\frac{1}{\Gamma(1+\varrho)} \int_{0}^{1} \Psi\left(\frac{1}{\frac{1}{\mathfrak{M}}+\frac{1}{\mathfrak{m}}-\left(\frac{1-\vartheta}{a}+\frac{\vartheta}{b}\right)}\right)(d \vartheta)^{\varrho},
\end{aligned}
$$

we infer that

$$
\begin{aligned}
\frac{1}{\Gamma(1+\varrho)} \Psi\left(\frac{1}{\frac{1}{\mathfrak{M}}+\frac{1}{\mathfrak{m}}-\frac{a+b}{2 a b}}\right) & \leq \frac{1}{\Gamma(1+\varrho)} \int_{0}^{1} \Psi\left(\frac{1}{\frac{1}{\mathfrak{M}}+\frac{1}{\mathfrak{m}}-\left(\frac{\vartheta}{a}+\frac{1-\vartheta}{b}\right)}\right)(d \vartheta)^{\varrho} \\
& \leq \frac{1}{2^{\varrho} \Gamma(1+\varrho)}\left[\Psi\left(\frac{1}{\frac{1}{\mathfrak{M}}+\frac{1}{\mathfrak{m}}-\frac{1}{a}}\right)+\Psi\left(\frac{1}{\frac{1}{\mathfrak{M}}+\frac{1}{\mathfrak{m}}-\frac{1}{b}}\right)\right]
\end{aligned}
$$

Since $\frac{1}{a_{l}}, \frac{1}{\bar{a}} \in[\mathfrak{m}, \mathfrak{M}]$, we can write

$$
\begin{aligned}
\Psi\left(\frac{1}{\frac{1}{\mathfrak{M}}+\frac{1}{\mathfrak{m}}-\frac{1}{2}\left(\frac{1}{\bar{a}}+\frac{1}{a_{l}}\right)}\right) & \leq \frac{\Gamma(1+\varrho)}{\left(\frac{1}{a_{l}}-\frac{1}{\bar{a}}\right) \varrho} \frac{1}{\frac{1}{\mathfrak{M}}+\frac{1}{\mathfrak{m}}-\frac{1}{\bar{a}}} \frac{I^{(\varrho)}}{\frac{1}{\mathfrak{M}}+\frac{1}{\mathfrak{m}}-\frac{1}{a_{l}}} \frac{\Psi(x)}{x^{2 \varrho}} \\
& \leq \frac{1}{2^{\varrho}}\left[\Psi\left(\frac{1}{\frac{1}{\mathfrak{M}}+\frac{1}{\mathfrak{m}}-\frac{1}{\bar{a}}}\right)+\Psi\left(\frac{1}{\frac{1}{\mathfrak{M}}+\frac{1}{\mathfrak{m}}-\frac{1}{a_{l}}}\right)\right]
\end{aligned}
$$

due to

$$
\frac{1}{\Gamma(1+\varrho)} \int_{0}^{1} \Psi\left(\frac{1}{\frac{1}{\mathfrak{M}}+\frac{1}{\mathfrak{m}}-\left(\frac{\vartheta}{\bar{a}}+\frac{1-\vartheta}{a_{l}}\right)}\right)(d \vartheta)^{\varrho}=\frac{1}{\left(\frac{1}{a_{l}}-\frac{1}{\bar{a}}\right)} \frac{1}{\frac{1}{\mathfrak{M}}+\frac{1}{\mathfrak{m}}-\frac{1}{a}} I_{\frac{(\varrho)}{\mathfrak{M}^{\prime}}+\frac{1}{\mathfrak{m}}-\frac{1}{a_{l}}} \frac{\Psi(x)}{x^{2 \varrho}} .
$$

Multiplying by $\gamma_{\imath}^{\varrho}>0(l=1, \ldots, s)$ and summing over $\imath$ from 1 to $s$, we may deduce

$$
\begin{aligned}
& \sum_{l=1}^{s} \gamma_{l}^{\varrho} \Psi\left(\frac{1}{\frac{1}{\mathfrak{M}}+\frac{1}{\mathfrak{m}}-\frac{1}{2}\left(\frac{1}{\bar{a}}+\frac{1}{a_{l}}\right)}\right) \\
& \quad \leq \sum_{l=1}^{s} \frac{\gamma_{l}^{\varrho} \Gamma(1+\varrho)}{\left(\frac{1}{a_{l}}-\frac{1}{\bar{a}}\right)^{\varrho}} \frac{1}{\frac{1}{\mathfrak{M}}+\frac{1}{\mathfrak{m}}-\frac{1}{\bar{a}}} I^{(\varrho)} \frac{1}{\mathfrak{M}+\frac{1}{\mathfrak{m}}-\frac{1}{a_{l}}} \frac{\Psi(x)}{x^{2 \varrho}} \\
& \quad \leq \frac{1}{2^{\varrho}}\left[\Psi\left(\frac{1}{\frac{1}{\mathfrak{M}}+\frac{1}{\mathfrak{m}}-\frac{1}{\bar{a}}}\right)+\sum_{l=1}^{s} \gamma_{l}^{\varrho} \Psi\left(\frac{1}{\frac{1}{\mathfrak{M}}+\frac{1}{\mathfrak{m}}-\frac{1}{a_{l}}}\right)\right] .
\end{aligned}
$$


On the other hand, by (G. J. I) for $\Psi \in G H K_{\ell}(I)$

$$
\begin{aligned}
\Psi\left(\frac{1}{\frac{1}{\mathfrak{M}}+\frac{1}{\mathfrak{m}}-\frac{1}{\bar{a}}}\right) & =\Psi\left(\frac{1}{\sum_{l=1}^{s} \gamma_{l}\left(\frac{1}{\mathfrak{M}}+\frac{1}{\mathfrak{m}}-\frac{1}{2}\left(\frac{1}{\bar{a}}+\frac{1}{a_{l}}\right)\right)}\right) \\
& \leq \sum_{l=1}^{s} \gamma_{l}^{\varrho} \Psi\left(\frac{1}{\frac{1}{\mathfrak{M}}+\frac{1}{\mathfrak{m}}-\frac{1}{2}\left(\frac{1}{\bar{a}}+\frac{1}{a_{l}}\right)}\right),
\end{aligned}
$$

and by Lemma 3 and (G. J. M. I) for $\Psi \in G H K_{\varrho}(I)$

$$
\begin{aligned}
\frac{1}{2^{\varrho}} & {\left[\Psi\left(\frac{1}{\frac{1}{\mathfrak{M}}+\frac{1}{\mathfrak{m}}-\frac{1}{\bar{a}}}\right)+\sum_{l=1}^{s} \gamma_{l}^{\varrho} \Psi\left(\frac{1}{\frac{1}{\mathfrak{M}}+\frac{1}{\mathfrak{m}}-\frac{1}{a_{l}}}\right)\right] } \\
& \leq \frac{1}{2^{\varrho}}\left[\Psi(\mathfrak{M})+\Psi(\mathfrak{m})-\sum_{j=1}^{s} \gamma_{j}^{\varrho} \Psi\left(a_{j}\right)+\Psi(\mathfrak{M})+\Psi(\mathfrak{m})-\sum_{l=1}^{s} \gamma_{l}^{\varrho} \Psi\left(a_{l}\right)\right] \\
& =\Psi(\mathfrak{M})+\Psi(\mathfrak{m})-\sum_{l=1}^{s} \gamma_{l}^{\varrho} \Psi\left(a_{l}\right) .
\end{aligned}
$$

Combining (11), (12), and (13), we get (10).

Remark 5 If we take $\varrho=1$ in Theorem 8, then it gives [[33], Theorem 2.6].

Remark 6 Let all the assumptions of Theorem 8 hold, then

$$
\begin{aligned}
& \Psi\left(\frac{1}{\sum_{l=1}^{s} \frac{\gamma_{l}}{a_{l}}}\right) \leq \sum_{l=1}^{s} \gamma_{l}^{\varrho} \Psi\left(\frac{2}{\sum_{l=1}^{s} \frac{\gamma_{l}}{a_{l}}+\frac{1}{a_{l}}}\right) \\
& \leq \sum_{l=1}^{s} \frac{\gamma_{l}^{\varrho} \Gamma(1+\varrho)}{\left(\sum_{l=1}^{s} \frac{\gamma_{l}}{a_{l}}-\frac{1}{a_{l}}\right) \varrho} \frac{1}{\sum_{l=1}^{s} \frac{\gamma}{l}_{l}} I_{a_{l}}^{(\varrho)} \frac{\Psi(x)}{x^{2 \varrho}} \\
& \leq \sum_{l=1}^{s} \gamma_{l}^{\varrho} \Psi\left(a_{l}\right) \text {. }
\end{aligned}
$$

\section{Related results}

In this section, we present the following related results for local fractional integrals.

Theorem 9 If $\Psi:[c, d] \subseteq(0, \infty) \longrightarrow \mathbb{R}^{\varrho}$ such that $\Psi \in G H K_{\varrho}(I)$ and $a_{1}, a_{2}, \ldots, a_{s} \in[c, d]$, then

$$
\begin{aligned}
& \Psi\left(\frac{2 a_{1} a_{2}}{a_{1}+a_{2}}\right)+\cdots+\Psi\left(\frac{2 a_{s-1} a_{s}}{a_{s-1}+a_{s}}\right)+\Psi\left(\frac{2 a_{s} a_{1}}{a_{s}+a_{1}}\right) \\
& \quad \leq \Psi\left(a_{1}\right)+\Psi\left(a_{s}\right)+\frac{(s-1)^{\varrho}}{s^{\varrho}} \sum_{l=1}^{s} \Psi\left(a_{l}\right)-\Psi\left(\frac{1}{\frac{1}{a_{1}}+\frac{1}{a_{s}}-\sum_{l=1}^{s} \frac{\frac{1}{s}}{a_{l}}}\right) .
\end{aligned}
$$


Proof From the generalized harmonic convexity of $\Psi$, we get

$$
\begin{aligned}
& \Psi\left(\frac{2 a_{1} a_{2}}{a_{1}+a_{2}}\right)+\cdots+\Psi\left(\frac{2 a_{s-1} a_{s}}{a_{s-1}+a_{s}}\right)+\Psi\left(\frac{2 a_{s} a_{1}}{a_{s}+a_{1}}\right) \\
& \quad \leq \frac{\Psi\left(a_{1}\right)+\Psi\left(a_{2}\right)}{2^{\varrho}}+\cdots+\frac{\Psi\left(a_{s-1}\right)+\Psi\left(a_{s}\right)}{2^{\varrho}}+\frac{\Psi\left(a_{s}\right)+\Psi\left(a_{1}\right)}{2^{\varrho}} \\
& \quad=\Psi\left(a_{1}\right)+\cdots+\Psi\left(a_{s}\right) \\
& \quad=\sum_{l=1}^{s} \Psi\left(a_{l}\right) .
\end{aligned}
$$

It is obvious that

$$
\begin{aligned}
\sum_{l=1}^{s} \Psi\left(a_{l}\right)= & \frac{(s-1)^{\varrho}}{s^{\varrho}} \sum_{l=1}^{s} \Psi\left(a_{l}\right)+\frac{1}{s^{\varrho}} \sum_{l=1}^{s} \Psi\left(a_{l}\right) \\
= & \Psi\left(a_{1}\right)+\Psi\left(a_{s}\right)+\frac{(s-1)^{\varrho}}{s^{\varrho}} \sum_{i=1}^{s} \Psi\left(a_{l}\right)-\left[\Psi\left(a_{1}\right)+\Psi\left(a_{s}\right)\right. \\
& \left.-\sum_{l=1}^{s}\left(\frac{1}{s}\right)^{\varrho} \Psi\left(a_{l}\right)\right] \\
\leq & \Psi\left(a_{1}\right)+\Psi\left(a_{s}\right)+\frac{(s-1)^{\varrho}}{s^{\varrho}} \sum_{i=1}^{s} \Psi\left(a_{l}\right)-\Psi\left(\frac{1}{\frac{1}{a_{1}}+\frac{1}{a_{s}}-\sum_{i=1}^{s} \frac{\frac{1}{s}}{a_{l}}}\right)
\end{aligned}
$$

Combining (15) and (16), we get (14).

Remark 7 If we take $\varrho=1$ in Theorem 9, then it reduces to Theorem 3.3 (see [16]).

Theorem 10 If $\Psi:[c, d] \subseteq(0, \infty) \longrightarrow \mathbb{R}^{\varrho}$ such that $\Psi \in G H K_{\varrho}(I)$ and $a_{1}, a_{2}, \ldots, a_{s} \in[c, d]$, then

$$
\sum_{i=1}^{s} \Psi\left(b_{l}\right) \leq \Psi\left(a_{1}\right)+\Psi\left(a_{s}\right)+\frac{(s-1)^{\varrho}}{s^{\varrho}} \sum_{i=1}^{s} \Psi\left(a_{l}\right)-\Psi\left(\frac{1}{\frac{1}{a_{1}}+\frac{1}{a_{s}}-\sum_{i=1}^{s} \frac{\frac{1}{s}}{a_{l}}}\right)
$$

where $b_{l}=\frac{1}{\frac{s-1}{s} \frac{1}{\varrho}+\frac{1}{s} \frac{1}{a_{l}}}$ and $\varrho=\frac{1}{\frac{1}{s} \sum_{l=1}^{s} \frac{1}{a_{l}}}$.

Proof Using (G. J. I) for $\Psi \in G H K_{\varrho}(I)$, we have

$$
\begin{aligned}
\sum_{i=1}^{s} \Psi\left(b_{l}\right) & =\Psi\left(b_{1}\right)+\cdots+\Psi\left(b_{n}\right) \\
& =\Psi\left(\frac{1}{\frac{s-1}{s} \frac{1}{\varrho}+\frac{1}{s} \frac{1}{a_{1}}}\right)+\cdots+\Psi\left(\frac{1}{\frac{s-1}{s} \frac{1}{\varrho}+\frac{1}{s} \frac{1}{a_{s}}}\right) \\
& \leq\left[\left(\frac{1}{s}\right)^{\varrho} \Psi(\varrho)+\left(\frac{s-1}{s}\right)^{\varrho} \Psi\left(a_{1}\right)\right]+\cdots+\left[\left(\frac{1}{s}\right)^{\varrho} \Psi(\varrho)+\left(\frac{s-1}{s}\right)^{\varrho} \Psi\left(a_{s}\right)\right]
\end{aligned}
$$




$$
\begin{aligned}
& =\Psi(\varrho)+\left(\frac{s-1}{s}\right)^{\varrho} \sum_{l=1}^{s} \Psi\left(a_{l}\right) \\
& =\Psi\left(\frac{1}{\frac{1}{s} \sum_{l=1}^{s} \frac{1}{a_{l}}}\right)+\left(\frac{s-1}{s}\right)^{\varrho} \sum_{l=1}^{s} \Psi\left(a_{l}\right) \\
& \leq\left(\frac{1}{s}\right)^{\varrho} \sum_{l=1}^{s} \Psi\left(a_{l}\right)+\left(\frac{s-1}{s}\right)^{\varrho} \sum_{l=1}^{s} \Psi\left(a_{l}\right) .
\end{aligned}
$$

Combining (16) and (18), we get (17).

Remark 8 If we take $\varrho=1$ in Theorem 10 , then it reduces to Theorem 3.4 (see [16]).

Theorem 11 Let $I=[\mathfrak{m}, \mathfrak{M}] \subseteq(0, \infty)$ be an interval. If $\Psi \in G H K_{\varrho}(I)$ on the interval $I$ with $\mathfrak{m}<\mathfrak{M}$ and for all $\zeta_{1}, \zeta_{2} \in[\mathfrak{m}, \mathfrak{M}]$. If $\Psi(x) \in I_{x}^{(\varrho)}[\mathfrak{m}, \mathfrak{M}]$, then

$$
\begin{aligned}
\Psi\left(\frac{1}{\frac{1}{\mathfrak{M}}+\frac{1}{\mathfrak{m}}-\frac{\zeta_{1}+\zeta_{2}}{2 \zeta_{1} \zeta_{2}}}\right) & \leq \Psi(\mathfrak{M})+\Psi(\mathfrak{m})-\frac{\zeta_{1}^{\varrho} \zeta_{2}^{\varrho}}{\left(\zeta_{2}-\zeta_{1}\right)^{\varrho}} \Gamma(1+\varrho)_{\zeta_{1}} I_{\zeta_{2}}^{(\varrho)} \frac{\Psi(x)}{x^{2 \varrho}} \\
& \leq \Psi(\mathfrak{M})+\Psi(\mathfrak{m})-\Psi\left(\frac{2 \zeta_{1} \zeta_{2}}{\zeta_{1}+\zeta_{2}}\right) .
\end{aligned}
$$

Proof Since $\Psi \in G H K_{\varrho}(I)$ on the interval $I=[\mathfrak{m}, \mathfrak{M}]$, setting $\vartheta=\frac{1}{2}$ in inequality (2), we have

$$
\Psi\left(\frac{1}{\frac{1}{\mathfrak{M}}+\frac{1}{\mathfrak{m}}-\frac{\overline{\zeta_{1}}+\overline{\zeta_{2}}}{2 \overline{\zeta_{1}} \overline{\zeta_{2}}}}\right) \leq \Psi(\mathfrak{M})+\Psi(\mathfrak{m})-\frac{\Psi\left(\overline{\zeta_{1}}\right)+\Psi\left(\bar{\zeta}_{2}\right)}{2^{\varrho}}
$$

for all $\bar{\zeta}_{1}, \bar{\zeta}_{2} \in[\mathfrak{m}, \mathfrak{M}]$. Choosing $\bar{\zeta}_{1}=\frac{\zeta_{1} \zeta_{2}}{\vartheta \zeta_{2}+(1-\vartheta) \zeta_{1}}, \bar{\zeta}_{2}=\frac{\zeta_{1} \zeta_{2}}{\vartheta \zeta_{1}+(1-\vartheta) \zeta_{2}}$ for all $\zeta_{1}, \zeta_{2} \in[\mathfrak{m}, \mathfrak{M}]$ and $\vartheta \in[0,1]$, we obtain

$$
\Psi\left(\frac{1}{\frac{1}{\mathfrak{M}}+\frac{1}{\mathfrak{m}}-\frac{\zeta_{1}+\zeta_{2}}{2 \zeta_{1} \zeta_{2}}}\right) \leq \Psi(\mathfrak{M})+\Psi(\mathfrak{m})-\frac{\Psi\left(\frac{\zeta_{1} \zeta_{2}}{\vartheta \zeta_{2}+(1-\vartheta) \zeta_{1}}\right)+\Psi\left(\frac{\zeta_{1} \zeta_{2}}{\vartheta \zeta_{1}+(1-\vartheta) \zeta_{2}}\right)}{2^{\varrho}}
$$

Multiplying by $\frac{1}{\Gamma(1+\varrho)}$ on both sides of the above equation and then integrating w.r.t $\vartheta$ over $[0,1]$, we have

$$
\begin{aligned}
\frac{1}{\Gamma(1+\varrho)} \Psi\left(\frac{1}{\frac{1}{\mathfrak{M}}+\frac{1}{\mathfrak{m}}-\frac{\zeta_{1}+\zeta_{2}}{2 \zeta_{1}}}\right) \\
\leq \frac{1}{\Gamma(1+\varrho)}[\Psi(\mathfrak{M})+\Psi(\mathfrak{m})]-\frac{1}{2 \varrho}\left[\frac{1}{\Gamma(1+\varrho)} \int_{0}^{1} \Psi\left(\frac{\zeta_{1} \zeta_{2}}{\vartheta \zeta_{2}+(1-\vartheta) \zeta_{1}}\right)(d \vartheta)^{\varrho}\right. \\
\left.\quad+\frac{1}{\Gamma(1+\varrho)} \int_{0}^{1} \Psi\left(\frac{\zeta_{1} \zeta_{2}}{\vartheta \zeta_{1}+(1-\vartheta) \zeta_{2}}\right)(d \vartheta)^{\varrho}\right] \\
=\frac{1}{\Gamma(1+\varrho)}[\Psi(\mathfrak{M})+\Psi(\mathfrak{m})]-\frac{1}{2^{\varrho}}\left(\frac{\zeta_{1} \zeta_{2}}{\zeta_{2}-\zeta_{1}}\right)^{\varrho}\left[\frac{1}{\Gamma(1+\varrho)} \int_{\zeta_{1}}^{\zeta_{2}} \frac{\Psi(x)}{x^{2 \varrho}}(d x)^{\varrho}\right. \\
\left.\quad+\frac{1}{\Gamma(1+\varrho)} \int_{\zeta_{1}}^{\zeta_{2}} \frac{\Psi(y)}{y^{2 \varrho}}(d y)^{\varrho}\right]
\end{aligned}
$$




$$
=\frac{1}{\Gamma(1+\varrho)}[\Psi(\mathfrak{M})+\Psi(\mathfrak{m})]-\frac{\zeta_{1}^{\varrho} \zeta_{2}^{\varrho}}{\left(\zeta_{2}-\zeta_{1}\right)^{\varrho}} \zeta_{1} I_{\zeta_{2}}^{(\varrho)} \frac{\Psi(x)}{x^{2 \varrho}}
$$

that is,

$$
\Psi\left(\frac{1}{\frac{1}{\mathfrak{M}}+\frac{1}{\mathfrak{m}}-\frac{\zeta_{1}+\zeta_{2}}{2 \zeta_{1} \zeta_{2}}}\right) \leq \Psi(\mathfrak{M})+\Psi(\mathfrak{m})-\frac{\zeta_{1}^{\varrho} \zeta_{2}^{\varrho}}{\left(\zeta_{2}-\zeta_{1}\right)^{\varrho}} \Gamma(1+\varrho)_{\zeta_{1}} I_{\zeta_{2}}^{(\varrho)} \frac{\Psi(x)}{x^{2 \varrho}}
$$

and so the first inequality of (19) is proved. If $\Psi \in G H K_{\varrho}(I)$, then for $\vartheta \in[0,1]$ we have

$$
\begin{aligned}
\Psi\left(\frac{2 \zeta_{1} \zeta_{2}}{\zeta_{1}+\zeta_{2}}\right) & =\Psi\left(\frac{1}{\frac{1}{2}\left(\frac{\vartheta}{\zeta_{1}}+\frac{(1-\vartheta)}{\zeta_{2}}+\frac{(1-\vartheta)}{\zeta_{1}}+\frac{\vartheta}{\zeta_{2}}\right)}\right) \\
& \leq \frac{1}{2^{\varrho}}\left[\Psi\left(\frac{\zeta_{1} \zeta_{2}}{\vartheta \zeta_{2}+(1-\vartheta) \zeta_{1}}\right)+\Psi\left(\frac{\zeta_{1} \zeta_{2}}{\vartheta \zeta_{1}+(1-\vartheta) \zeta_{2}}\right)\right]
\end{aligned}
$$

Multiplying by $\frac{1}{\Gamma(1+\varrho)}$ on both sides of the above equation and then integrating w.r.t $\vartheta$ over $[0,1]$, we get

$$
\Psi\left(\frac{2 \zeta_{1} \zeta_{2}}{\zeta_{1}+\zeta_{2}}\right) \leq \frac{\zeta_{1}^{\varrho} \zeta_{2}^{\varrho}}{\left(\zeta_{2}-\zeta_{1}\right)^{\varrho}} \Gamma(1+\varrho)_{\zeta_{1}} I_{\zeta_{2}}^{(\varrho)} \frac{\Psi(x)}{x^{2 \varrho}}
$$

and then

$$
-\Psi\left(\frac{2 \zeta_{1} \zeta_{2}}{\zeta_{1}+\zeta_{2}}\right) \geq-\frac{\zeta_{1}^{\varrho} \zeta_{2}^{\varrho}}{\left(\zeta_{2}-\zeta_{1}\right)^{\varrho}} \Gamma(1+\varrho)_{\zeta_{1}} I_{\zeta_{2}}^{(\varrho)} \frac{\Psi(x)}{x^{2 \varrho}}
$$

Adding $\Psi(\mathfrak{M})+\Psi(\mathfrak{m})$ to both sides of the above equation, we have

$$
\Psi(\mathfrak{M})+\Psi(\mathfrak{m})-\Psi\left(\frac{2 \zeta_{1} \zeta_{2}}{\zeta_{1}+\zeta_{2}}\right) \geq \Psi(\mathfrak{M})+\Psi(\mathfrak{m})-\frac{\zeta_{1}^{\varrho} \zeta_{2}^{\varrho}}{\left(\zeta_{2}-\zeta_{1}\right)^{\varrho}} \Gamma(1+\varrho)_{\zeta_{1}} I_{\zeta_{2}}^{(\varrho)} \frac{\Psi(x)}{x^{2 \varrho}}
$$

Combining (20) and (21), we get the second inequality of (19).

Remark 9 If we take $\varrho=1$ in Theorem 11, then it gives [[16], Theorem 3.5].

\section{Application to special means}

Let $\zeta_{1}<\zeta_{2}$ and $\zeta_{1}, \zeta_{2} \in \mathbb{R}^{\varrho}$ considering the following $\varrho$-type special means.

(1) The generalized arithmetic mean:

$$
A_{\varrho}\left(\zeta_{1}, \zeta_{2}\right)=\frac{\zeta_{1}^{\varrho}+\zeta_{2}^{\varrho}}{2^{\varrho}}
$$

(2) The generalized $\xi$-logarithmic mean:

$$
L_{\xi \varrho}\left(\zeta_{1}, \zeta_{2}\right)=\left[\frac{\Gamma(1+\xi \varrho)}{\Gamma(1+(\xi+1) \varrho)} \frac{\zeta_{2}^{(\xi+1) \varrho}-\zeta_{1}^{(\xi+1) \varrho}}{\left(\zeta_{2}-\zeta_{1}\right)^{\varrho}}\right]^{1 / \xi},
$$

where $\xi \in \mathbb{Z} \backslash\{-1,0\}, \zeta_{1}, \zeta_{2} \in \mathbb{R}, \zeta_{1} \neq \zeta_{2}$.

(3) The generalized geometric mean:

$$
G_{\varrho}\left(\zeta_{1}, \zeta_{2}\right)=\left(\zeta_{1}^{\varrho} \zeta_{2}^{\varrho}\right)^{\frac{1}{2}}
$$


(4) The generalized harmonic mean:

$$
H_{\varrho}\left(\zeta_{1}, \zeta_{2}\right)=\frac{\left(2 \zeta_{1} \zeta_{2}\right)^{\varrho}}{\zeta_{1}^{\varrho}+\zeta_{2}^{\varrho}}
$$

Consider the mapping $\Psi:(0, \infty) \rightarrow \mathbb{R}^{\varrho}, \Psi(x)=\frac{\Gamma(1+k \varrho)}{\Gamma(1+(k+1) \varrho} x^{(k+1) \varrho}, x>0, k \geq 1$. Then $\left|\Psi^{\varrho}(x)\right|^{q}=x^{k q \varrho}$, where $q \geq 1$ is a generalized harmonic convex function on $(0, \infty)$.

Proposition 1 For $\mathfrak{m}, \mathfrak{M}, \zeta_{1}, \zeta_{2} \in \mathbb{R}, \zeta_{1}, \zeta_{2} \in[\mathfrak{m}, \mathfrak{M}], 0<\mathfrak{m}<\mathfrak{M}$, and $k>1$, we have

$$
\left[\frac{1}{2^{\varrho} A_{\varrho}\left(\mathfrak{m}^{-1}, \mathfrak{M}^{-1}\right)-H_{\varrho}^{-1}\left(\zeta_{1}, \zeta_{2}\right)}\right]^{k+1} \leq 2^{\varrho} A_{\varrho}\left(\mathfrak{m}^{k+1}, \mathfrak{M}^{k+1}\right)-A_{\varrho}\left(\zeta_{1}^{k+1}, \zeta_{2}^{k+1}\right) ;
$$

and

$$
\begin{aligned}
& \left(\frac{1}{2^{\varrho} A_{\varrho}\left(\mathfrak{m}^{-1}, \mathfrak{M}^{-1}\right)-H_{\varrho}^{-1}\left(\zeta_{1}, \zeta_{2}\right)}\right)^{k+1} \\
& \quad \leq \Gamma(1+\varrho) L_{-(k+1) \varrho}^{-(k+1)}\left(2 A\left(\mathfrak{m}^{-1}, \mathfrak{M}^{-1}\right)-\zeta_{1}^{-1}, 2 A\left(\mathfrak{m}^{-1}, \mathfrak{M}^{-1}\right)-\zeta_{2}^{-1}\right) \\
& \quad \leq 2^{\varrho} A_{\varrho}\left(\mathfrak{m}^{k+1}, \mathfrak{M}^{k+1}\right)-A_{\varrho}\left(\zeta_{1}^{k+1}, \zeta_{2}^{k+1}\right) .
\end{aligned}
$$

Proof Let $s=2, \gamma_{1}=\gamma_{2}=\frac{1}{2}, a_{1}=\zeta_{1}, a_{2}=\zeta_{2}$, and $\Psi(x)=\frac{\Gamma(1+k \varrho)}{\Gamma(1+(k+1) \varrho} x^{(k+1) \varrho}$, where $x>0, k>1$ in Theorem 6 and Corollary 1 lead to the desired inequality (22) and (23) immediately respectively.

\section{Applications to probability density functions (P.D.F)}

Consider a random variable $\chi$ with generalized P.D.F $\tau:[\mathfrak{m}, \mathfrak{M}] \longrightarrow\left[0^{\varrho}, 1^{\varrho}\right]$ i.e. $\tau \in$ $G H K_{\varrho}([\mathfrak{m}, \mathfrak{M}])$ possessing the cumulative distribution function

$$
P_{\varrho}(\chi \leq z)=F_{\varrho}(z)=\frac{1}{\Gamma(1+\varrho)} \int_{\mathfrak{m}}^{z} \tau(\varsigma)(d \varsigma)^{\varrho} .
$$

The generalized expectation

$$
E_{\varrho}=\frac{1}{\Gamma(1+\varrho)} \int_{\mathfrak{m}}^{\mathfrak{M}} \varsigma^{\varrho} \tau(\varsigma)(d \varsigma)^{\varrho} .
$$

It is easy to see that

$$
E_{\varrho}(\chi)=\mathfrak{M}^{\varrho}-\frac{1}{\Gamma(1+\varrho)} \int_{\mathfrak{m}}^{\mathfrak{M}} F_{\varrho}(\varsigma)(d \varsigma)^{\varrho} .
$$

The generalized expectation $E_{p \varrho}$ is a $p$-moment, $p \in \mathbb{R} \backslash\{-1,0\}$ is

$$
E_{p \varrho}=\frac{1}{\Gamma(1+\varrho)} \int_{\mathfrak{m}}^{\mathfrak{M}} \varsigma^{p \varrho} \tau(\varsigma)(d \varsigma)^{\varrho} .
$$

Define the generalized $(-\varrho)$-moment by

$$
E_{-\varrho}=\frac{1}{\Gamma(1+\varrho)} \int_{\mathfrak{m}}^{\mathfrak{M}} \frac{1}{\varsigma^{\varrho}} \tau(\varsigma)(d \varsigma)^{\varrho} .
$$

For more details, see $[34,35]$. 
The following two propositions can be obtained by using the cumulative distribution function and generalized $(-\varrho)$-moment of the random variable $\chi$ from our results associated with Sects. 3 and 4.

Proposition 2 Let $s=2, \gamma_{1}=\gamma_{2}=\frac{1}{2}, a_{1}=\zeta_{1}$, and $a_{2}=\zeta_{2}$ in Theorem 6, we have

$$
P_{\varrho}\left(\chi \leq \frac{1}{\frac{1}{\mathfrak{M}}+\frac{1}{\mathfrak{m}}-\frac{\zeta_{1}+\zeta_{2}}{2 \zeta_{1} \zeta_{2}}}\right) \leq P_{\varrho}(\chi \leq \mathfrak{M})+P_{\varrho}(\chi \leq \mathfrak{m})-\frac{P_{\varrho}\left(\chi \leq \zeta_{1}\right)+P_{\varrho}\left(\chi \leq \zeta_{2}\right)}{2^{\varrho}}
$$

Proposition 3 Let $s=2, \gamma_{1}=\gamma_{2}=\frac{1}{2}, a_{1}=\mathfrak{m}$, and $a_{2}=\mathfrak{M}$ in Corollary 1 , we have

$$
\begin{aligned}
P_{\varrho}\left(\chi \leq \frac{2 \mathfrak{m} \mathfrak{M}}{\mathfrak{m}+\mathfrak{M}}\right) & \leq \frac{\mathfrak{m}^{\varrho} \mathfrak{M} \mathfrak{M}^{\varrho}}{(\mathfrak{M}-\mathfrak{m})^{\varrho}} \frac{\Gamma(1+\varrho) \Gamma(1-2 \varrho)}{\Gamma(1-\varrho)}\left[\left(\frac{1}{\mathfrak{M}}\right)^{\varrho}-E_{-\varrho}(\chi)\right] \\
& \leq \frac{P_{\varrho}(\chi \leq \mathfrak{M})+P_{\varrho}(\chi \leq \mathfrak{m})}{2^{\varrho}} .
\end{aligned}
$$

\section{Conclusion}

In this paper, for the first time, we introduce Jensen's inequality for harmonic convex functions by using local fractional calculus. As a result we also introduce a variant of generalized Jensen-Mercer inequality in the fractal sense. Then we present the main lemma involving local fractional integrals. By using this main lemma, we establish generalized Jensen-Mercer-type inequalities on fractal sets $\mathbb{R}^{\varrho}(0<\varrho \leq 1)$. Moreover, an improvement and generalization of some results related to the class of generalized harmonically convex function via local fractional integrals are established. Using these inequalities, we obtain some generalized related results on the fractal space. Lastly, some applications to some special means of real numbers and probability density function are established. It is quite open to investigate Jensen and Jensen-type inequalities for other classes of generalized convex functions in the fractal sense. Since there is a massive body of literature about refinements, converses, and reverses of such Jensen and Jensen-type inequalities, so their generalized variants using local fractional calculus can be revealed also. One of the important directions is to give fractal Jensen-Mercer-Ostrowski type inequalities and give applications to continuous random variables and improve quadrature rules. Since local fractional calculus is quite effective from an application point of view, a bridge between inequality theory and their applications can be established, which may eventually generate optimal solutions.

\section{Acknowledgements}

The first authors is partially supported by Ministerio de Ciencia, Innovación y Universidades grant number PGC2018-097198-B-I00 and Fundación Séneca de la Región de Murcia grant number 20783/PI/18.

\section{Funding}

The first authors is partially supported by Ministerio de Ciencia, Innovación y Universidades grant number

PGC2018-097198-B-I00 and Fundación Séneca de la Región de Murcia grant number 20783/PI/18.

\section{Availability of data and materials}

The data used to support the findings of this study are included within the article.

\section{Declarations}

Competing interests

The authors declare that they have no competing interests. 
Authors' contributions

All authors contributed equally to this article. All authors read and approved the final manuscript.

\section{Author details}

'Department of Mathematics, COMSATS University Islamabad, Lahore Campus, Pakistan. ${ }^{2}$ Department of Mathematics, Anand International College of Engineering, Jaipur, 303012, Rajasthan, India. ${ }^{3}$ Nonlinear Dynamics Research Center (NDRC), Ajman University, Ajman AE 346, United Arab Emirates. ${ }^{4}$ Departamento de Matematica Aplicada y Estadistica, Universidad Politecnica de Cartagena, Hospital de Marina, Murcia 30203, Spain. ${ }^{5}$ Department of Mathematics, Faculty of Science, King Abdulaziz University, P.O. Box 80203, Jeddah 21589, Saudi Arabia.

\section{Publisher's Note}

Springer Nature remains neutral with regard to jurisdictional claims in published maps and institutional affiliations.

\section{Received: 15 May 2021 Accepted: 10 December 2021 Published online: 03 January 2022}

\section{References}

1. Mitrinović, D.S., Pečarić, J.E., Fink, A.M.: Classical and New Inequalities in Analysis. Mathematics and Its Applications (East European Series), vol. 61. Kluwer Academic, Dordrecht (1993)

2. Dragomir, S.S., Pearce, C.E.M.: Selected Topics on Hermite-Hadamard Inequalities and Applications. RGMIA Monographs, Victoria University (2000)

3. Kalsoom, H., Latif, M.A., Rashid, S., Baleanu, D., Chu, Y.M.: New $(p, q)$-estimates for different types of integral inequalities via $(\alpha, m)$-convex mappings. Open Math. 18(1), 1830-1854 (2020)

4. Shi, H.N., Zhang, J.: Some new judgement theorems of Schur geometric and Schur harmonic convexities for a class of symmetric functions. J. Inequal. Appl. 2013, Article ID 527 (2013)

5. Işcan, I.: Hermite-Hadamard type inequalities for harmonically convex functions. Hacet. J. Math. Stat. 43(6), 935-942 (2014)

6. Latif, M.A., Dragomir, S.S., Momoniat, E.: Some Fejér type inequalities for harmonically convex functions with applications to special means. Int. J. Anal. Appl. 13(1), 1-14 (2016)

7. Jung, C.Y., Yussouf, M., Chu, Y.M., Farid, G., Kang, S.M.: Generalized fractional Hadamard and Fejér-Hadamard inequalities for generalized harmonically convex functions. J. Math. 2020, Article ID 8245324 (2020)

8. Sababheh, M.: Improved Jensen's inequality. Math. Inequal. Appl. 20(2), 389-403 (2017)

9. Khan, S., Khan, M.A., Butt, S.I., Chu, Y.M.: A new bound for the Jensen gap pertaining twice differentiable functions with applications. Adv. Differ. Equ. 2020, Article ID 333 (2020)

10. Khan, S., Khan, M.A., Chu, Y.M.: New converses of Jensen inequality via Green functions with applications. Rev. R. Acad. Cienc. Exactas Fís. Nat., Ser. A Mat. 114(3), 14 (2020)

11. Mehmood, N., Butt, S.I., Pečarić, D., Pečarić, J.: Generalizations of cyclic refinements of Jensen's inequality by Lidstone's polynomial with applications in information theory. J. Math. Inequal. 14(1), 249-271 (2019)

12. Khan, S., Khan, M.A., Chu, Y.M.: Converses of the Jensen inequality derived from the Green functions with applications in information theory. Math. Methods Appl. Sci. 43(5), 2577-2587 (2020)

13. Butt, S.I., Bakula, M.K., Pečarić, D., Pečarić, J.: Jensen-Grüss inequality and its applications for the Zipf-Mandelbrot law. Math. Methods Appl. Sci. 44(2), 1664-1673 (2021)

14. Mercer, A.McD: A variant of jensens inequality. J. Inequal. Pure Appl. Math. 4(4), Article ID 73 (2003)

15. Dragomir, S.S.: Inequalities of Jensen type for HA-convex functions. An. Univ. Oradea, Fasc. Mat. 27, 103-124 (2020)

16. Baloch, I.A., Mughal, A.A., Chu, Y.M., Haq, A.U., Sen, M.D.L.: A variant of Jensen-type inequality and related results for harmonic convex functions. AIMS Math. 5(6), 6404-6418 (2020)

17. Gunerhan, H., Celik, E.: Analytical and approximate solutions of fractional partial differential algebraic equations. Appl. Math. Nonlinear Sci. 5(1), 109-119 (2020)

18. Ihan, E., Krymaz, I.: A generalization of truncated M-fractional derivative and applications to fractional differential equations. Appl. Math. Nonlinear Sci. 5(1), 171-188 (2020)

19. Kaur, D., Agarwal, P., Rakshi, M., Chand, M.: Fractional calculus involving (p, q)-Mathieu type series. Appl. Math. Nonlinear Sci. 5(2), 15-34 (2020)

20. Gençoǧlu, M.T., Agarwal, P.: Use of quantum differential equations in sonic processes. Appl. Math. Nonlinear Sci. 1 , 1-8 (2019)

21. Butt, S.I., Umar, M., Rashid, S., Akdemir, A.O., Chu, Y.M.: New Hermite-Jensen-Mercer-type inequalities via k-fractional integrals. Adv. Differ. Equ. 2020(1), 1 (2020)

22. Yang, X.J.: Advanced Local Fractional Calculus and Its Applications. World Science Publisher, New York (2012)

23. Ziane, D., Cherif, M.H., Cattani, C., Belghaba, K.: Yang-Laplace decomposition method for nonlinear system of local fractional partial differential equations. Appl. Math. Nonlinear Sci. 4(2), 489-502 (2019)

24. Mandelbrot, B.B.: The Fractal Geometry of Nature. Macmillan, New York (1983)

25. Carpinteri, A., China, B., Cornetti, P.: Static-kinematic duality and the principle of virtual work in the mechanics of fractal media. Comput. Methods Appl. Mech. Eng. 191, 3-19 (2001)

26. Zhao, Y., Cheng, D.F., Yang, X.J.: Approximation solutions for local fractional schr ordinger equation in the one-dimensional cantorian system. Adv. Math. Phys. 2013, 5 (2013)

27. Mo, H., Sui, X., Yu, D.: Generalized convex functions and some inequalities on fractal sets (2014). 1404.3964. arXiv preprint

28. Sun, W.: Generalized harmonically convex functions on fractal sets and related Hermite-Hadamard type inequalities. J. Nonlinear Sci. Appl. 10(11), 5869-5880 (2017)

29. Noor, M.A., Noor, K.I., Iftikhar, S., Awan, M.U.: Fractal integral inequalities for harmonic convex functions. Appl. Math. Inf. Sci. 12(4), 831-839 (2018)

30. Sun, W., Liu, Q.: Hadamard type local fractional integral inequalities for generalized harmonically convex functions and applications. Math. Methods Appl. Sci. 43(9), 5776-5787 (2020) 
31. Iftikhar, S., Erden, S., Kumam, P., Awan, M.U.: Local fractional Newton's inequalities involving generalized harmonic convex functions. Adv. Differ. Equ. 2020, Article ID 185 (2020)

32. Chu, Y.M., Rashid, S., Abdeljawad, T., Khalid, A., Kalsoom, H.: On new generalized unified bounds via generalized exponentially harmonically s-convex functions on fractal sets. Adv. Differ. Equ. 218, 33 (2021)

33. Baloch, I.A., Mughal, A.A., Chu, Y.M., Haq, A.U., Sen, M.D.L.: Improvement and generalization of some results related to the class of harmonically convex functions and applications. J. Math. Comput. Sci. 22(2021), 282-294 (2010)

34. Erden, S., Sarikaya, M.Z., Celik, N.: Some generalized inequalities involving local fractional integrals and their applications for random variables and numerical integration. J. Appl. Math. Stat. Inform. 12(2), 49-65 (2016)

35. Barnett, N.S., Dragomir, S.S.: Some inequalities for random variables whose probability density functions are bounded using a pre-Grüss inequality. Kyungpook Math. J. 40(2), 299-311 (2000)

Submit your manuscript to a SpringerOpen ${ }^{\circ}$ journal and benefit from:

- Convenient online submission

- Rigorous peer review

- Open access: articles freely available online

High visibility within the field

- Retaining the copyright to your article

Submit your next manuscript at $\boldsymbol{\triangleright}$ springeropen.com 